\title{
Molecular Characterization of the FCoV-like Canine Coronavirus HLJ-071 in China
}

\author{
Zhige Tian \\ University of Yibin \\ Miaomiao Zheng \\ University of Yibin \\ Ying Deng \\ University of Yibin \\ Dandan Gou \\ University of Yibin \\ Peng Guo \\ University of Yibin \\ Feng Cong \\ Guangdong Laboratory Animals Monitoring Institute \\ Xiaoliang Hu ( $\sim$ liang679@163.com ) \\ University of Yibin
}

\section{Research Article}

Keywords: canine coronavirus, transcription-regulating sequence, recombination, cell tropism

Posted Date: February 23rd, 2021

DOl: https://doi.org/10.21203/rs.3.rs-222737/v1

License: (c) (i) This work is licensed under a Creative Commons Attribution 4.0 International License.

Read Full License

Version of Record: A version of this preprint was published at BMC Veterinary Research on November 27th, 2021. See the published version at https://doi.org/10.1186/s12917-021-03073-8. 


\section{Abstract \\ Background}

According to differences in antigens and genetic composition, canine coronavirus (CCoV) consists of two genotypes, CCoV- $\$ and CCoV- $\rrbracket$. Recently, CCoVs with mutant variations have been found to be pantropic and pathogenic in dogs.

\section{Results}

In this study, we isolated a CCoV, designated HLJ-071, from a dead 5-week-old female Welsh Corgi with severe diarrhea and vomiting. Sequence analysis suggested that HLJ-071 bears a complete ORF3abc when compared with classic CCoV isolates (1-71, K378 and S378). In addition, a variable region was located between the $S$ gene and the open reading frames (ORF) 3a gene, in which HLJ-071 has a deletion of 104 nucleotides (nts) when compared with classic CCoV strains 1-71, S378 and K378. Phylogenetic analysis based on the $S$ gene and complete sequences showed that HLJ-071 is closely related to Feline Coronavirus (FCoV) II. Recombination analysis suggested that HLJ-071 originated from the recombination of FCoV 79-1683, FCoV DF2 and CCoV A76. Finally, cell tropism experiments suggested that HLJ-071 is able to replicate in canine macrophages/monocytes.

\section{Conclusion}

The present study involved the isolation and genetic characterization of a variant CCoV strain. The spike protein and ORF3abc of CCoV might play a key role in viral tropism, which could affect replication in monocyte/macrophage cells. This will provide essential information for further understanding the evolution of CCoV in China.

\section{Background}

COVID-19 is once again bringing coronaviruses back into focus. Coronaviruses, which belong to the family Coronaviridae, order Nidovirales, are single-stranded positive-sense RNA viruses, which have been widely detected in wild animals [1], domesticated animals [2, 3], humans [4]and pets [5].

Currently, coronaviruses can be divided into four subfamilies, named Alpha, Beta, Gamma and Delta [6]. Canine coronavirus (CCoV) is a member of the alpha subfamily of coronaviruses which, based on the spike protein gene, has been divided into two distinct genotypes, CCoV I and CCoV II [7, 8], both of which are distributed widely [9-14]. Two different subtypes, CCoV Ila and CCoV Ilb, have been found in dogs. CCoV Ila has served as the classic CCoV strain; it causes mild enteritis in young dogs [15]. CCoV IIb emerged because of homologous recombination between the transmissible gastroenteritis virus of swine (TGEV) and CCoV Ila strains [16]; it causes acute gastroenteritis and the virus can be detected in the 
intestines and other internal organs [16]. An intermediate virus, CCoV-A76, which possess a distinct spike which confers pathogenicity and is the result of recombination between CCoV I and CCoV II, has been detected [17].

Recently, more virulent CCoV strains have been reported without obvious coinfections [18-20]. In these infections, variant viruses with novel recombinant and deletion forms have been detected in the intestines and other organs, resulting in new pathogenic viruses [21, 22]. In addition, HLJ-073, with deletion of ORF3abc, has altered cell tropism and can replicate effectively in canine macrophages/monocytes and human myeloid leukemia mononuclear cells (THP-1 cells) [21]. Therefore, CCoV is of concern for public health.

In this study, we isolated the HLJ-071 strain of CCoV from canine fecal samples in China. To better understand the genetic characterization of a variant CCoV strain, the complete genome sequence, phylogenetic tree and cell tropism were investigated. This will provide essential information for further understanding the evolution of CCoV in China.

\section{Methods}

\section{Clinical case}

During the summer of 2015, a 5-week-old female Welsh Corgi with severe diarrhea and vomiting was submitted after death for laboratory investigation. Post-mortem examination of the dog showed hemorrhagic enteritis and hemorrhages on the surface of the lungs.

\section{Isolation and purification}

Rapid diagnosis kits were employed to identify general canine viral pathogens, including canine distemper virus (CDV), canine parvovirus (CPV), canine adenovirus-1 (CAV-1), CAV-2 and CCoV (Bionote, Hwaseong-si, Gyeonggi-do, South Korea). The primers P-F and P-R were employed as described [21].

Crandell feline kidney (CrFK) cells were grown in D-MEM supplemented with $10 \%$ fetal calf serum (FCS). The fecal sample was homogenized in phosphate-buffered saline (PBS) and centrifuged at 3,000 $\mathrm{g}$ for 15 min. Following this, the supernatant was filtered through a $0.22-\mu \mathrm{m}$ pore size filter and inoculated into CrFK cells, which had formed confluent monolayers. When the sample was passaged three times, cytopathic effects (CPE) were observed. After three rounds of purification by plaque assay [23], the purified virus was titrated and harvested by one cycle of freezing and thawing, and aliquots were stored at $-80^{\circ} \mathrm{C}$.

\section{Electron microscopy}

The electron microscopy protocol for negative-stain and thin-section examination was described previously $[24,25]$. 


\section{Isolation and culture of canine blood monocytes}

Canine blood monocytes were isolated following a previously described protocol [26]. Briefly, canine blood monocytes were isolated from five specific-pathogen-free (SPF) dogs. The blood mononuclear cells were purified on Histopaque-1077 (Sigma-Aldrich), and then seeded in a 24-well dish and cultured at 37 ${ }^{\circ} \mathrm{C}$ with $5 \% \mathrm{CO}_{2}$. After $24 \mathrm{~h}$, nonadherent cells were removed and washed twice with PBS buffer.

\section{Indirect immunofluorescence assay (IFA)}

The IFA was conducted using a standard procedure. Briefly, mononuclear cells were inoculated with CCoV isolates at a multiplicity of infection (MOI) of 1 for $48 \mathrm{~h}$ and the supernatant removed; after washing with PBS, the infected cells were fixed with paraformaldehyde (4\%) for 30 min. After blocking with $2 \%$ BSA for $2 \mathrm{~h}$ at $37^{\circ} \mathrm{C}$, the cells were incubated with $\mathrm{N}$ protein polyclonal antibodies $(1: 400)$ for $1 \mathrm{~h}$ at $37^{\circ} \mathrm{C}$ [27], followed by a fluorescein isothiocyanate-conjugated goat anti-mouse antibody against immunoglobulin G (1:1000; Abcam, UK). The CCoV-N polyclonal antibody was prepared in our laboratory [21].

\section{Genome sequencing and phylogeny analysis}

Fourteen pairs of primers were designed, based on the conserved regions of CCoV strain HLJ-073 [21]. The RNA extraction and cDNA synthesis were performed as previously described [24].

Sequence data were assembled and analyzed using Clustal X software (1.83), Vector 10 and DNASTAR. Phylogenetic trees based on the complete sequences and the spike proteins were produced using the neighbor-joining $(\mathrm{NJ})$ method with the Kimura two-parameter model in molecular evolutionary genetics analysis (MEGA) software (version 4.0). The support for the tree nodes was calculated with 1,000 replicates. All of the sequence information were listed in Table 1. Simplot 3.5.1 was used to evaluate the recombination events between the reference CCoV and FCoV strains. The HLJ-071 sequence obtained in this study was assembled and submitted to the GenBank database under accession number KY063616.

\section{Results}

\section{Viral isolation and identification}

The results of the colloidal gold diagnostic reagent and PCR confirmed that the fecal sample was CCoVpositive; the sample was negative for CPV, CAV and CDV (data not shown). After inoculation of CrFK cells with samples and three serial passages, one CCoV isolate, designated HLJ-071, was obtained from the fecal samples; CPE were found in the CrFK cells $3-5$ days post-inoculation, with rounding and detachment of cells into the medium (Fig. 1A, 1B). The titre of HLJ-071 was $10^{7.5}$ median tissue culture infective dose $\left(\mathrm{TCID}_{50}\right) / \mathrm{mL}$ in CrFK cells. Electron microscopy showed that the virus had a circular shape with petal-shaped protrusions, and had a diameter of about $150 \mathrm{~nm}$ (Fig. 1C). Ultra-thin sections of infected CrFK cells displayed typical virus particles in the cytoplasm (Fig. 1D). 
The complete genome sequence of HLJ-071 was assembled and comparative analysis with other canine coronavirus was performed. The full genome of HLJ-071 was amplified using the 14 pairs of primers referenced to HLJ-073. The complete sequence of HLJ-071 was 29,319 nucleotides (nts) in length, including the 5'non-translated region (NTR)-ORF1-S-ORF3abc-E-M-N-ORF7ab-3'poly A tail. The 5'portion NTR of the genome contained a 230-nt NTR, ORF1a $(231-12,287)$ and ORF1ab $(231-20,057)$. Four structural proteins, S, E, M and N, were found to be encoded by ORF S (20,284-24,648 nt), ORF E $(25,826-26,074 \mathrm{nt})$, ORF M $(26,055-26,876 \mathrm{nt})$ and ORF N (26,889-28,037 nt). Five non-structural protein-encoding genes were ORF3a (24,712-24,948 nt), ORF3b (24,893-25,108 nt), ORF3c $(25,105-$ $25,860 \mathrm{nt})$, ORF7a (28,042-28,347 nt) and ORF7b (28,352-28,993 nt).

Sequence analysis suggested that there was an entire ORF3abc with 1,149 nts when compared with classic CCoV strains 1-71 and K378. However, a variable region was located between the S gene and ORF 3a gene, in which HLJ-071 had a deletion of 104 nts when compared with classic CCoV strains 171, S378 and K378 (Fig. 2). In addition, variant CCoV strains, feline enteric coronavirus (FECV), feline infectious peritonitis virus (FIPV) and TGEV also bear a deletion, to varying degrees, when compared with classical CCoVs. A highly conserved core sequence (CS), 5'-CUAAAC-3', is present in HLJ-071 and variant CCoV, FECV, FIPV and TGEV strains. There is a $\mathrm{C}$ to $\mathrm{T}$ mutation in classical CCoVs, resulting in a different CS, 5'-UUAAAC-3'. All of these findings suggest that the transcription of ORF3abc is influenced by these variable regions.

In the phylogenetic analysis based on complete sequences, HLJ-071 fell into the FCoV cluster, close to TN-449, HLJ-073 and A76. The complete spike protein did not cluster with either type I or type II CCoVs, and was related to FCoV WSU 79-1683. In addition, analysis of the S1 (receptor-binding) domain showed that it clustered closely with FCoV 79-1683 and HLJ-073, while the S2 (fusion) domain clustered with CCoV Ilb 174/06 and FCoV WSU79-1683 (Fig. 3). The occurrence of recombination was detected in HLJ071, involving CCoV A76, FCoV 79-1683 and DF2, which has led to the emergence of a new genotype of FCoV-like CCoVs (Fig. 4).

\section{Cell tropism of HLJ-071}

Previous studies showed that ORF3abc deletion in canine and feline coronavirus alters the cell tropism $[26,27]$. To investigate further the in vitro growth characteristics of HLJ-071, canine monocytes were inoculated with HLJ-071 and HLJ-073 at an MOI of 1. The results showed that HLJ-073 can replicate efficiently in canine monocytes; however, HLJ-071 could replicate only poorly in these cells (Fig. 5). Furthermore, the titers of HLJ-071 and HLJ-073 were determined at 24 h.p.i. in CrFK cell lines and canine monocytes. The results showed that the growth characteristics of HLJ-071 were similar to those of HLJ073 and reached $10^{7.5} \mathrm{TCID}_{50} / \mathrm{mL}$ at $24 \mathrm{~h}$ p.i. in CrFK cells (Fig. 6A). However, the titer of HLJ-071 peaked at $2 \times 10^{1.6} \mathrm{TCID}_{50} / \mathrm{mL}$ at $6 \mathrm{~h}$ p.i., showing significantly slower growth when compared with the titer of HLJ-073, which reached $10^{3.5} \mathrm{TCID}_{50} / \mathrm{mL}$ at 36 h.p.i. (Fig. 6).

\section{Discussion}


Generally, CCoV causes intestinal infections, resulting in viral enteritis and diarrhea in dogs. In recent decades, however, an increasing number of pantropic strains have been reported to cause systemic infections with multiple organ damage [28]. The mechanism for the change in tissue tropism of CCoVs from enteropathogenic to systemic infection is unknown.

In this study, we isolated a CCoV, HLJ-071, from a dead 5-week-old female Welsh Corgi without apparent coinfections. Phylogenetic analysis based on the complete sequence suggested that HLJ-071 is close to FCoV II and distinct from other CCoV I and II strains. In terms of the major structural protein spike protein, HLJ-071 was closely related to FCoV WSU79-1683 and domestic strain HLJ-073, different from other Chinese strains B135/JS/2018, B194/GZ/2019, B639/ZJ/2019, B203/GZ/2019 and B447/ZJ/2019. HLJ-071 recombining with CCoV A76, FCoV 79-1683 and DF2, formed a unique cluster with HLJ-073 and TN-449 between the classical CCoVs and FCoVs. All of these findings suggest that domestic CCoV strains were derived from different ancestors and have co-circulated in China and undergone evolution.

The cell tropism of FCoVs has been well investigated. Previous studies have indicated that the spike protein and ORF3abc play a crucial part in infection of macrophages by FCoVs [26, 29]. Feline infectious peritonitis viruses (FIPV) bearing partial deletion of ORF3abc are able to replicate in cells of the monocyte/macrophage lineage and then disseminate to the organs, causing systemic infections [30]. In contrast, feline enteric coronavirus (FECV) with entire ORF3abc primarily replicates in enterocytes and is unable to replicate in cells of the monocyte/macrophage lineage. Among CCoVs, a systemic infection involving CCoV CB/05, with a partial deletion of ORF3b and a FCoV-like spike protein, was found in 2005 [31]. We speculate that this stain could replicate in monocyte/macrophage cells, although no data were available from viral tropism experiments. Furthermore, we have isolated a CCoV strain, HLJ-073, bearing a 350-nt deletion in ORF3abc and a FCoV-like spike protein, and reported that it can replicate efficiently in canine monocyte/macrophage cells and human THP-1 cells [21]. In this study, HLJ-071, bearing the entire ORF3abc and FCoV spike protein, could replicate only weakly in canine monocyte/macrophage cells when compared with HLJ-073, which was distinct from the cell tropism of FECV. These findings suggest that 1) both HLJ-071 and HLJ-073 have a common feature, the acquisition of macrophage tropism; 2) deletion of ORF3abc can enhance replication in monocyte/macrophage cells, as shown by comparison of HLJ-071 and HLJ-073.

Previous studies have indicated that the $5^{\prime}$ and $3^{\prime}$ flanks of the transcription regulatory sequences (TRSs) of TGEV influence transcription levels (accumulation) and protein expression [32]. Interestingly, when comparing classic CCoVs, variant CCoVs, FECV, FIPV and TGEV, sequence analysis suggests that there is a variable region forward of the TRS, between the end of spike gene and ORF3a gene, which may influence the level and stability of RNA transcription and gene expression. Among the CCoV, FCoV and TGEV, a C to T mutant only found in the conserved CS of classic CCoVs K378 and 1-71, which may speculate that the transcription of subgenome was affected. Both of these findings suggest that, except for the function of the ORF3abc protein of $\mathrm{CCoV}$, the variable region may also contribute to alterations in cell tropism of CCoV. Further experiments are needed to investigate the mechanisms involved between the transcription of the subgenome and the function of ORF3abc in viral tropism. 


\section{Conclusion}

In this study, we indicated that: 1) CCoV HLJ-071 is closely related to FCoVs and shows recombination between CCoVs and FCoVs, indicating that CCoVs have undergone a rapid evolution in China. 2) The cell tropism of CCoVs may be correlated with the function of ORF3abc and transcription of the subgenome.

\section{Abbreviations}

Feline Coronavirus (FCoV); Canine coronavirus (CCoV); transmissible gastroenteritis virus (TGEV); human myeloid leukemia mononuclear cells (THP-1 cells); canine distemper virus (CDV); canine parvovirus (CPV); canine adenovirus-1 (CAV-1); Crandell feline kidney (CrFK); fetal calf serum (FCS); cytopathic effects (CPE); neighbor-joining ( $\mathrm{NJ}$ ); molecular evolutionary genetics analysis (MEGA); core sequence (CS); Feline infectious peritonitis viruses (FIPV); transcription regulatory sequences (TRSs); multiplicity of infection (MOI); median tissue culture infective dose (TCID50)

\section{Declarations}

\section{Ethics approval and consent to participate}

The present study was approved by the Animal Ethics Committee of Yibin University, Yibin, China, according to the OIE standards for use of animals in research and education. No animals were sacrificed specifically for this study. The dog was collected by the veterinary hospital. The owner of dog took the initiative to send the sick animal to the veterinary hospital for treatment. When the dog's condition worsens until death, and with the client's consent for further research, the body of the dogs were sent to University of Yibin. We reported the results of death of the dogs to the veterinary hospital according to the diagnostic findings, and we were not involved in the collection of samples.

\section{Consent for publication}

Not applicable.

\section{Availability of data and materials}

DNA sequences obtained in this study have been submitted to the GenBank database (accession number: KY063616).

\section{Competing interests}

The authors declare no competing interests.

\section{Funding}

This work was supported by the Doctor Launch Project of Yibin University (No. 2019QD09 and No. 2019QD10). The funders had no role in the study design, data analysis, and decision to publish, or 
preparation of the manuscript.

Authors' contribution

HXL designed the study, TZG, ZMM, DY and GDD performed the experiments. TZG drafted the manuscript. GP, CF and HXL revised the draft. All authors revised and approved the paper for publication.

\section{Acknowledgments}

Not applicable.

\section{References}

1. Yang XL, Hu B, Wang B, Wang MN, Zhang Q, Zhang W, Wu LJ, Ge XY, Zhang YZ, Daszak P et al: Isolation and Characterization of a Novel Bat Coronavirus Closely Related to the Direct Progenitor of Severe Acute Respiratory Syndrome Coronavirus. 2015, 90(6):3253-3256.

2. Jung K, Saif LJ, Wang Q: Porcine epidemic diarrhea virus (PEDV): An update on etiology, transmission, pathogenesis, and prevention and control. Virus research 2020, 286:198045.

3. McCluskey BJ, Haley C, Rovira A, Main R, Zhang Y, Barder S: Retrospective testing and case series study of porcine delta coronavirus in U.S. swine herds. Preventive veterinary medicine 2016, 123:185-191.

4. Morfopoulou S, Brown JR, Davies EG, Anderson G, Virasami A, Qasim W, Chong WK, Hubank M, Plagnol V, Desforges $\mathrm{M}$ et al: Human Coronavirus OC43 Associated with Fatal Encephalitis. The New England journal of medicine 2016, 375(5):497-498.

5. Tekes G, Thiel HJ: Feline Coronaviruses: Pathogenesis of Feline Infectious Peritonitis. Advances in virus research 2016, 96:193-218.

6. Carstens EB: Ratification vote on taxonomic proposals to the International Committee on Taxonomy of Viruses (2009). Archives of virology 2010, 155(1):133-146.

7. Decaro N, Buonavoglia C: An update on canine coronaviruses: viral evolution and pathobiology. Veterinary microbiology 2008, 132(3-4):221-234.

8. Le Poder S: Feline and canine coronaviruses: common genetic and pathobiological features. Advances in virology 2011, 2011:609465.

9. Costa EM, de Castro TX, Bottino Fde O, Garcia Rde C: Molecular characterization of canine coronavirus strains circulating in Brazil. Veterinary microbiology 2014, 168(1):8-15.

10. Cavalli A, Desario C, Kusi I, Mari V, Lorusso E, Cirone F, Kumbe I, Colaianni ML, Buonavoglia D, Decaro $\mathrm{N}$ : Detection and genetic characterization of Canine parvovirus and Canine coronavirus strains circulating in district of Tirana in Albania. Journal of veterinary diagnostic investigation: official publication of the American Association of Veterinary Laboratory Diagnosticians, Inc 2014, 26(4):563-566. 
11. Licitra BN, Whittaker GR, Dubovi EJ, Duhamel GE: Genotypic characterization of canine coronaviruses associated with fatal canine neonatal enteritis in the United States. Journal of clinical microbiology 2014, 52(12):4230-4238.

12. Pratelli A, Decaro N, Tinelli A, Martella V, Elia G, Tempesta M, Cirone F, Buonavoglia C: Two genotypes of canine coronavirus simultaneously detected in the fecal samples of dogs with diarrhea. Journal of clinical microbiology 2004, 42(4):1797-1799.

13. Decaro N, Mari V, Elia G, Lanave G, Dowgier G, Colaianni ML, Martella V, Buonavoglia C: Full-length genome analysis of canine coronavirus type I. Virus research 2015, 210:100-105.

14. Wang X, Li C, Guo D, Wang X, Wei S, Geng Y, Wang E, Wang Z, Zhao X, Su M et al: Co-Circulation of Canine Coronavirus I and Ila/b with High Prevalence and Genetic Diversity in Heilongjiang Province, Northeast China. PloS one 2016, 11(1):e0146975.

15. Decaro N, Buonavoglia C: Canine coronavirus: not only an enteric pathogen. The Veterinary clinics of North America Small animal practice 2011, 41(6):1121-1132.

16. Decaro N, Mari V, Campolo M, Lorusso A, Camero M, Elia G, Martella V, Cordioli P, Enjuanes L, Buonavoglia C: Recombinant canine coronaviruses related to transmissible gastroenteritis virus of Swine are circulating in dogs. Journal of virology 2009, 83(3):1532-1537.

17. Regan AD, Millet JK, Tse LP, Chillag Z, Rinaldi VD, Licitra BN, Dubovi EJ, Town CD, Whittaker GR: Characterization of a recombinant canine coronavirus with a distinct receptor-binding (S1) domain. Virology 2012, 430(2):90-99.

18. Decaro N, Martella V, Elia G, Campolo M, Desario C, Cirone F, Tempesta M, Buonavoglia C: Molecular characterisation of the virulent canine coronavirus CB/05 strain. Virus research 2007, 125(1):54-60.

19. Zappulli V, Caliari D, Cavicchioli L, Tinelli A, Castagnaro M: Systemic fatal type II coronavirus infection in a dog: pathological findings and immunohistochemistry. Research in veterinary science 2008, 84(2):278-282.

20. Escutenaire $S$, Isaksson M, Renström LH, Klingeborn B, Buonavoglia $C$, Berg M, Belák S, Thorén P: Characterization of divergent and atypical canine coronaviruses from Sweden. Archives of virology 2007, 152(8):1507-1514.

21. Chen S, Liu D, Tian J, Kang H, Guo D, Jiang Q, Liu J, Li Z, Hu X: Molecular characterization of HLJ073, a recombinant canine coronavirus strain from China with an ORF3abc deletion. 2019, 164(8):2159-2164.

22. Ntafis V, Mari V, Decaro N, Papanastassopoulou M, Papaioannou N, Mpatziou R, Buonavoglia C, Xylouri $\mathrm{E}$ : Isolation, tissue distribution and molecular characterization of two recombinant canine coronavirus strains. Veterinary microbiology 2011, 151(3-4):238-244.

23. Tuchiya K, Kasaoka T, Azetaka M, Takahashi E, Konishi S: Plaque assay for canine coronavirus in CRFK cells. Nihon juigaku zasshi The Japanese journal of veterinary science 1987, 49(3):571-573.

24. Hu X, Jr., Li N, Jr., Tian Z, Jr., Yin X, Jr., Qu L, Qu J: Molecular characterization and phylogenetic analysis of transmissible gastroenteritis virus $\mathrm{HX}$ strain isolated from China. $B M C$ veterinary research 2015, 11:72. 
25. Li Z, Shao Y, Liu C, Liu D, Guo D, Qiu Z, Tian J, Zhang X, Liu S, Qu L: Isolation and pathogenicity of the mammalian orthoreovirus MPC/04 from masked civet cats. Infection, genetics and evolution: journal of molecular epidemiology and evolutionary genetics in infectious diseases 2015, 36:55-61.

26. Bálint Á, Farsang A, Zádori Z, Hornyák Á, Dencso L, Almazán F, Enjuanes L, Belák S: Molecular characterization of feline infectious peritonitis virus strain DF-2 and studies of the role of ORF3abc in viral cell tropism. Journal of virology 2012, 86(11):6258-6267.

27. Chen S, Liu D, Tian J, Kang H, Guo D, Jiang Q, Liu J, Li Z, Hu X: Molecular characterization of HLJ073, a recombinant canine coronavirus strain from China with an ORF3abc deletion. Archives of virology 2019, 164(8):2159-2164.

28. Buonavoglia C, Decaro N, Martella V, Elia G, Campolo M, Desario C, Castagnaro M, Tempesta M: Canine coronavirus highly pathogenic for dogs. Emerging infectious diseases 2006, 12(3):492-494.

29. Rottier PJ, Nakamura K, Schellen P, Volders H, Haijema BJ: Acquisition of macrophage tropism during the pathogenesis of feline infectious peritonitis is determined by mutations in the feline coronavirus spike protein. Journal of virology 2005, 79(22):14122-14130.

30. Kipar A, May H, Menger S, Weber M, Leukert W, Reinacher M: Morphologic features and development of granulomatous vasculitis in feline infectious peritonitis. Veterinary pathology 2005, 42(3):321330 .

31. Decaro N, Mari V, von Reitzenstein M, Lucente MS, Cirone F, Elia G, Martella V, King VL, Di Bello A, Varello $\mathrm{K}$ et al: A pantropic canine coronavirus genetically related to the prototype isolate $\mathrm{CB} / 05$. Veterinary microbiology 2012, 159(1-2):239-244.

32. Alonso S, Izeta A, Sola I, Enjuanes L: Transcription regulatory sequences and mRNA expression levels in the coronavirus transmissible gastroenteritis virus. Journal of virology 2002, 76(3):1293-1308.

\section{Table}

Table 1. Source of sequences used in the experiment 


\begin{tabular}{|c|c|c|c|c|}
\hline Isolate & Type & Accession no. & Origin & Collection date \\
\hline $\mathrm{CB} / 05$ & CCoV IIa & KP981644 & ITA & 2005 \\
\hline $1-71$ & CCoV IIa & JQ404409 & GER & 1971 \\
\hline K378 & CCoV IIa & KC175340 & USA & 1978 \\
\hline TGEV-HX & I & KC962433 & $\mathrm{CHN}$ & 2013 \\
\hline BGF-10 & CCoV IIa & AY342160 & UK & 2002 \\
\hline INSAVC-1 & CCoV IIa & D13096 & UK & 1992 \\
\hline A76 & CCoV II & JN856008 & USA & 1976 \\
\hline Purdue & l & DQ811789 & USA & 2011 \\
\hline $23 / 03$ & CCoV I & KP849472 & ITA & 2004 \\
\hline Black & FCoV I & EU186072 & USA & 1980 \\
\hline WSU79-1683 & FCoV II & JN634064 & USA & 1979 \\
\hline TN449 & CCoV IIa & JQ404410 & USA & $1980 s$ \\
\hline DF2 & FCoV II & JQ408981 & USA & 1981 \\
\hline $79-1146$ & FCoV II & DQ010921 & USA & 1979 \\
\hline 171 & CCoV IIa & KC175339 & GER & 1971 \\
\hline HCOV 229E & l & AF 304460 & I & 1963 \\
\hline S378 & CCoV IIa & KC175341 & USA & 1978 \\
\hline GZ43 & l & EF192155 & $\mathrm{CHN}$ & 2003 \\
\hline KUK-HL & FCoV II & AB781789 & JPN & 2014 \\
\hline $450 / 07$ & CCoV IIb & GU146061 & ITA & 2012 \\
\hline FC1 & CCoV IIa & AB781790 & JPN & 2014 \\
\hline M91-267 & FCoV II & AB781788 & JPN & 2013 \\
\hline $\mathrm{RM}$ & FCoV I & FJ938051 & USA & 2002 \\
\hline NTU156 & FCoV II & GQ152141 & $\mathrm{CHN}$ & 2007 \\
\hline NTU336 & CCoV II & GQ477367 & $\mathrm{CHN}$ & 2008 \\
\hline $174 / 06$ & CCoV II & EU856362 & ITA & 2006 \\
\hline $430 / 07$ & CCoV II & EU924790 & ITA & 2007 \\
\hline $68 / 09$ & CCoV IIb & HQ450377 & GRE & 2009 \\
\hline $341 / 05$ & CCoV II & EU856361 & ITA & 2005 \\
\hline
\end{tabular}




\begin{tabular}{c|c|c|c|c} 
66/09 & CCoV IIb & HQ450376 & GRE & 2009 \\
$119 / 08$ & CCoV II & EU924791 & ITA & 2008 \\
UU16 & FCoV I & FJ938058 & NED & 2007 \\
UU21 & FCoV I & HQ012369 & NED & 2007 \\
UU23 & FCoV I & GU553362 & NED & 2007 \\
UU2 & FCoV I & FJ938060 & USA & 1993 \\
CATDAY28 & FCoV I & KU215428 & BEL & 2013 \\
UCD11a & FCoV I & FJ917519 & USA & 2008 \\
DM95/2003 & FbCoV & EF192156 & CHN & 2007 \\
HCM47 & CCoV II & LC190907 & VNM & 2019 \\
B639 ZJ 2019 & CCoV II & MT114538 & CHN & 2019 \\
B194 GZ 2019 & CCoV II & MT114543 & CHN & 2019 \\
B203 GZ 2019 & CCoV II & MT114542 & CHN & 2019 \\
B447 ZJ 2019 & CCoV II & MT114540 & CHN & 2019 \\
B135 JS 2018 & CCoV II & MT114544 & CHN & 2019 \\
\hline
\end{tabular}

Figures 

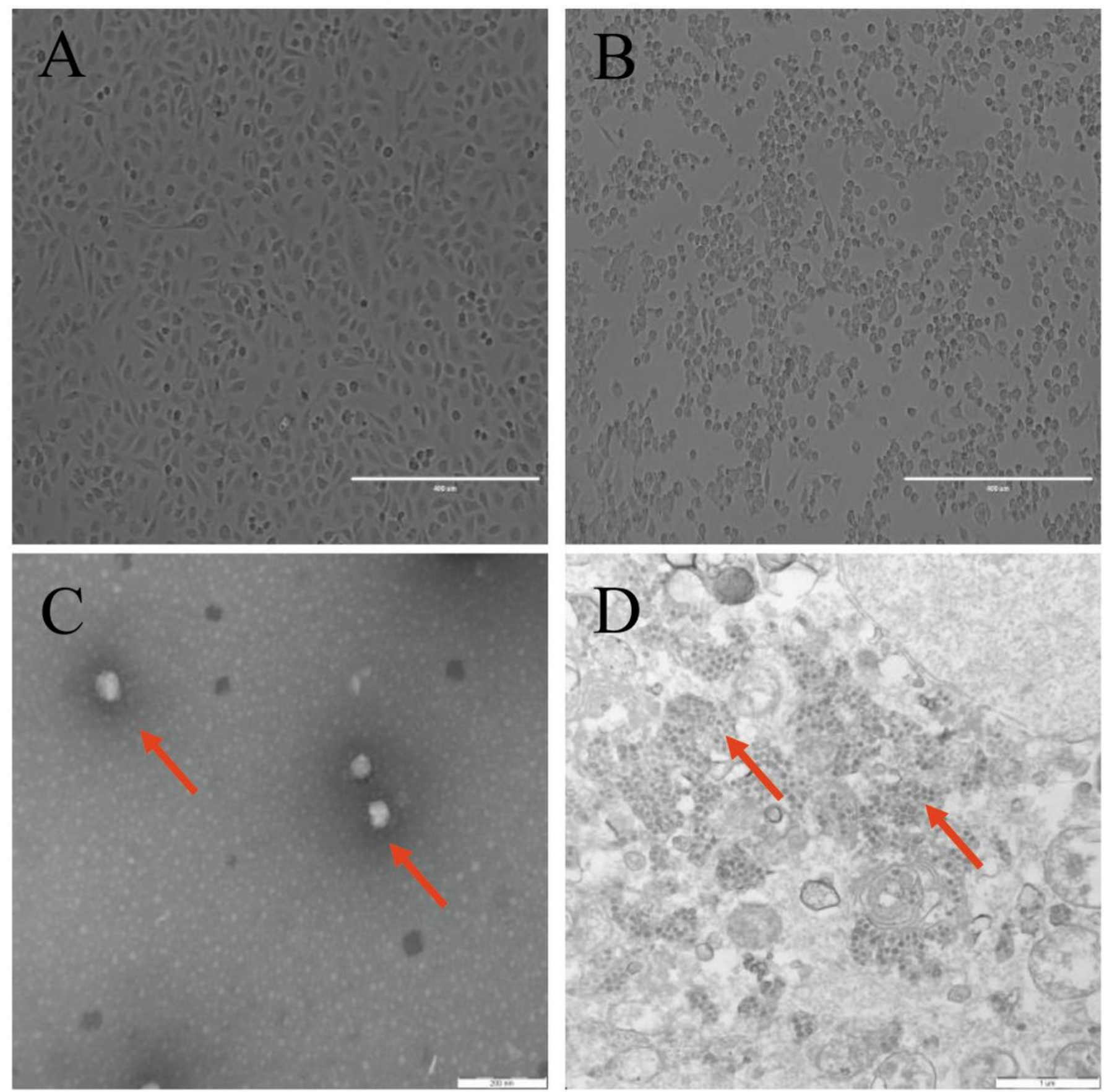

\section{Figure 1}

(A) Control (uninfected) CrFK cells. (B) Cytopathic effect (CPE) induced by HLJ-071 in CRrFK cells. (C) Electron micrograph of HLJ-071 negatively stained with $2 \%$ phosphotungstic acid. The scale bar represents $200 \mathrm{~nm}$. (D) Ultra-thin sections of CrFK cells infected with HLJ-071 display the typical particles in the cytosol.

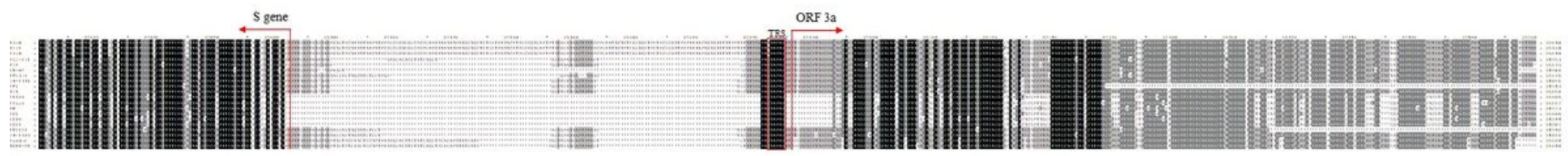


Figure 2

Multiple sequence alignment of the partial S and ORF3a of CCoV strains.

A

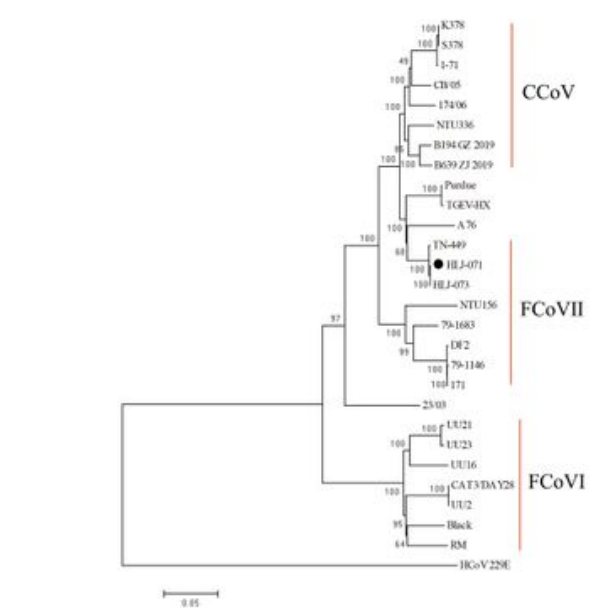

B

B

에

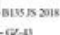

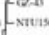

-kexsa

(tiss

$\operatorname{lin}_{n=2}$

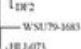

- imari

$\mathrm{C}$
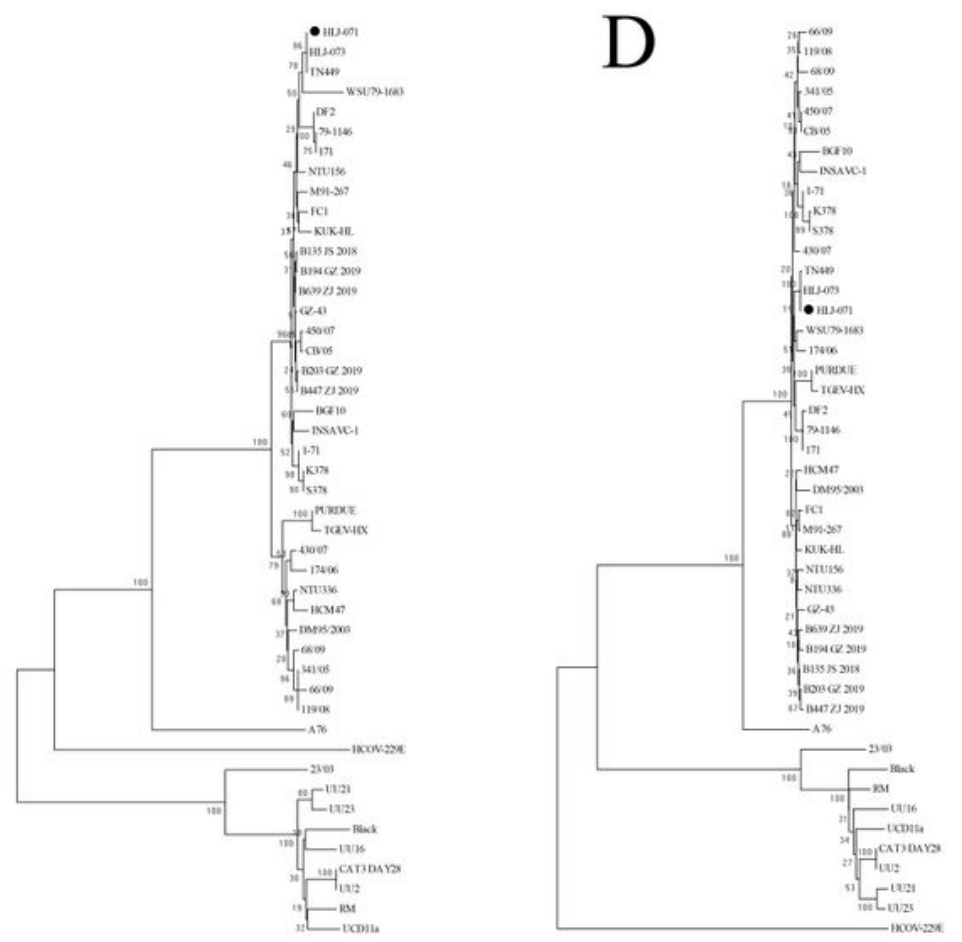

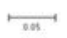

\section{Figure 3}

Phylogenetic analysis of the complete sequences, spike protein (S), S1, and S2 genome regions of HLJ071. Neighbor-joining was used for construction of the phylogenetic tree, with bootstrap values of 1000 
replicates shown at the branches. The scale bar represents the p-distance.

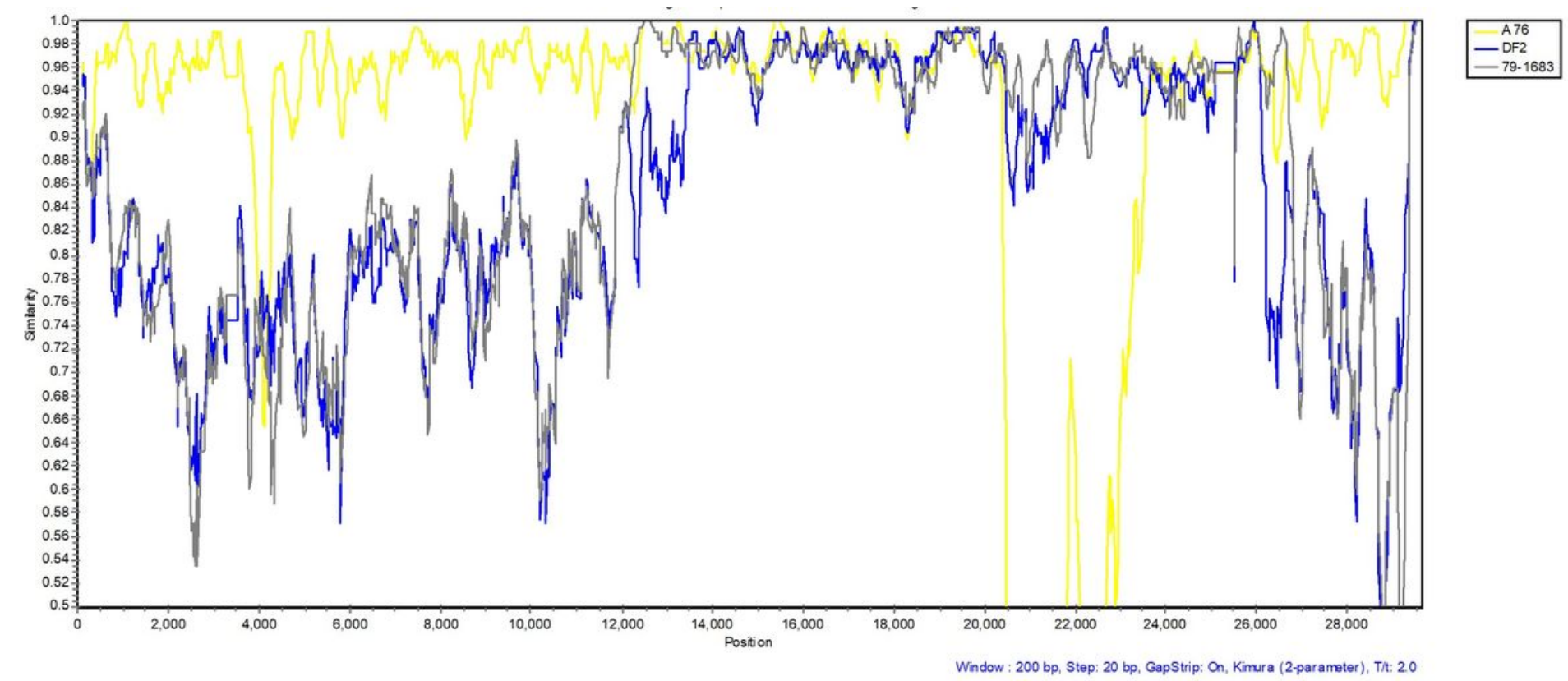

Figure 4

Similarity plot of the complete nucleotide sequences of CCoV HLJ-071 and three reference CCoV strains, FCoV DF2, FCoV WSU79-1683 and CCoV A76. The other parameters used included the Kimura (twoparameter) distance model, $2.0 \mathrm{Ts} / \mathrm{Tv}$ ratio, neighbor-joining tree model, and 1000 bootstrap replicates. 
Bright
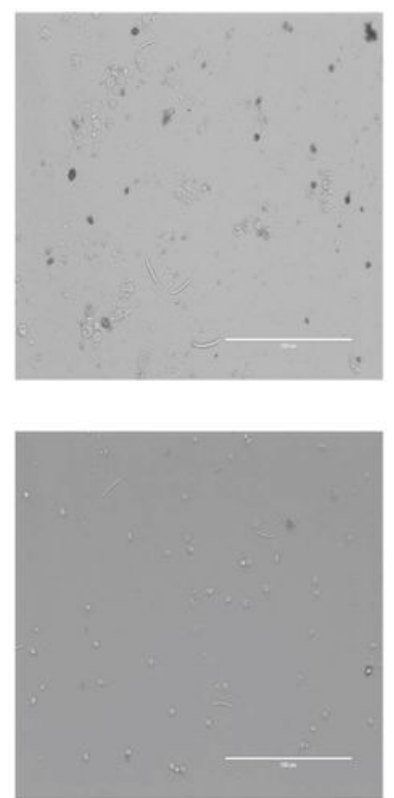

HLJ-071

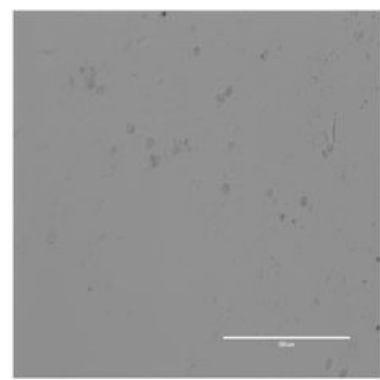

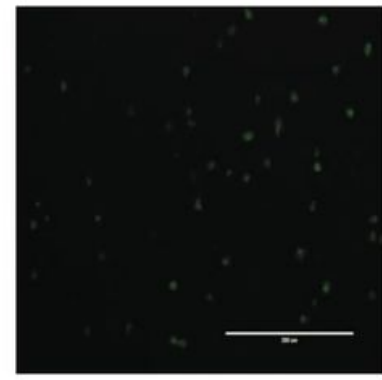

FITC
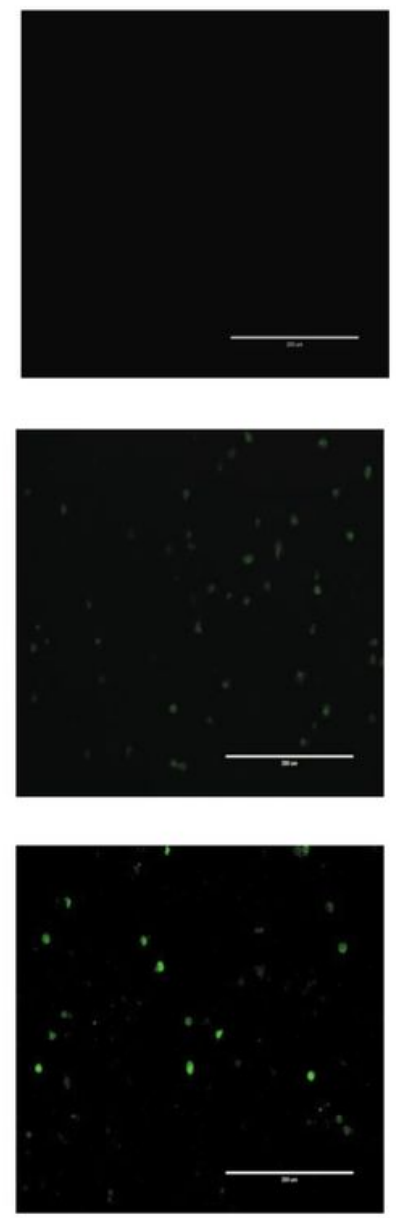
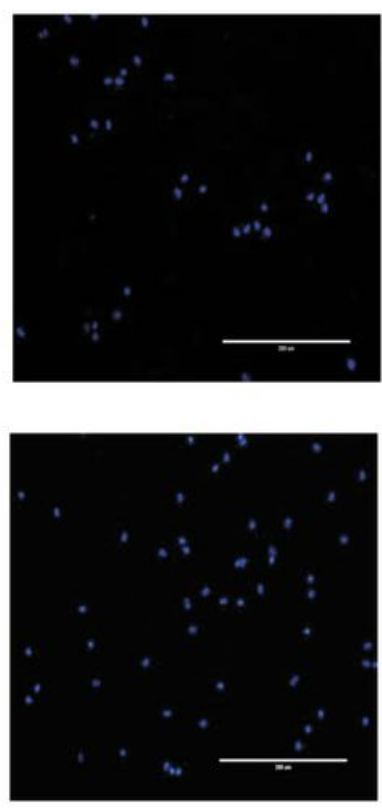

DAPI

Merge
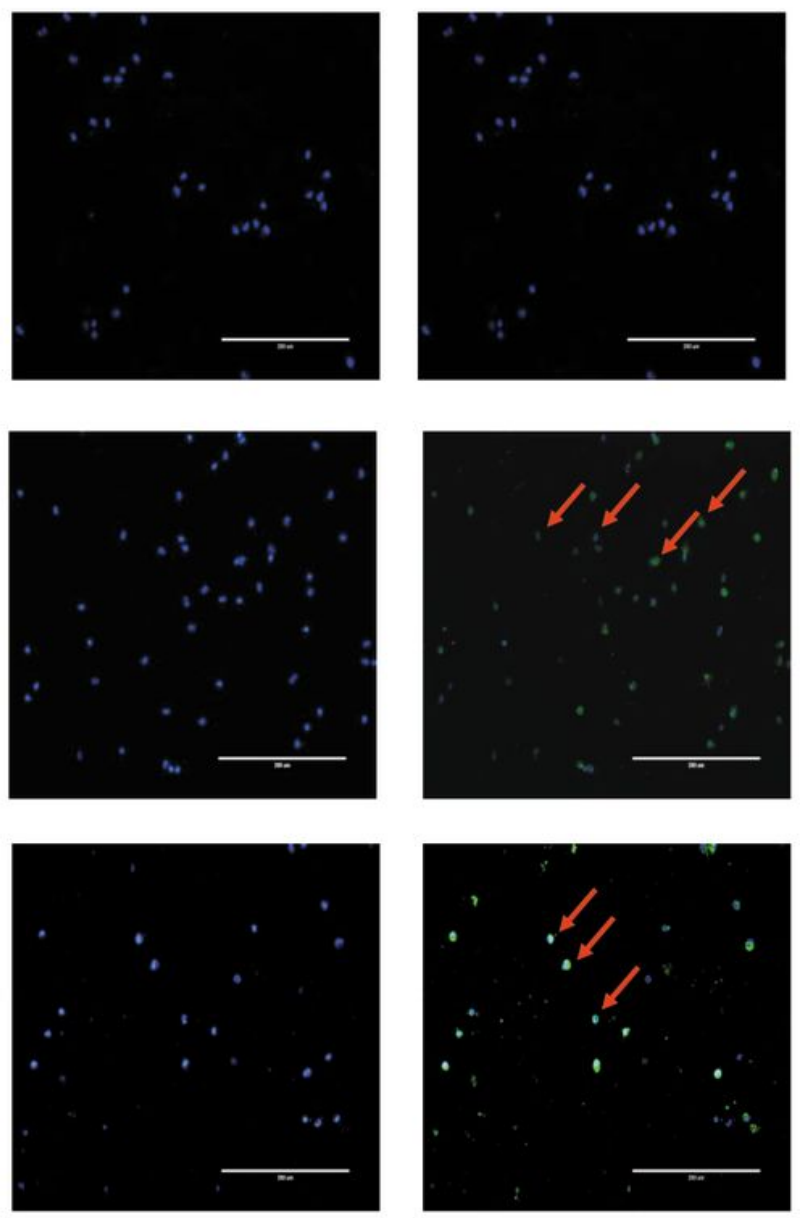

Figure 5

Indirect immunofluorescence detection of CCoVs in canine macrophages/monocytes. The cells were infected with HLJ-071 or HLJ-073 (MOI = 1) and detected using CCoV N protein-positive serum.

A

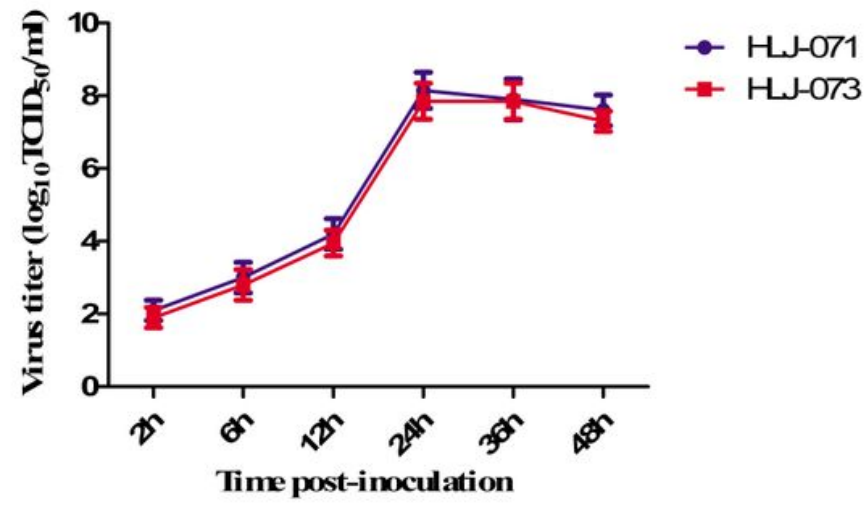

B

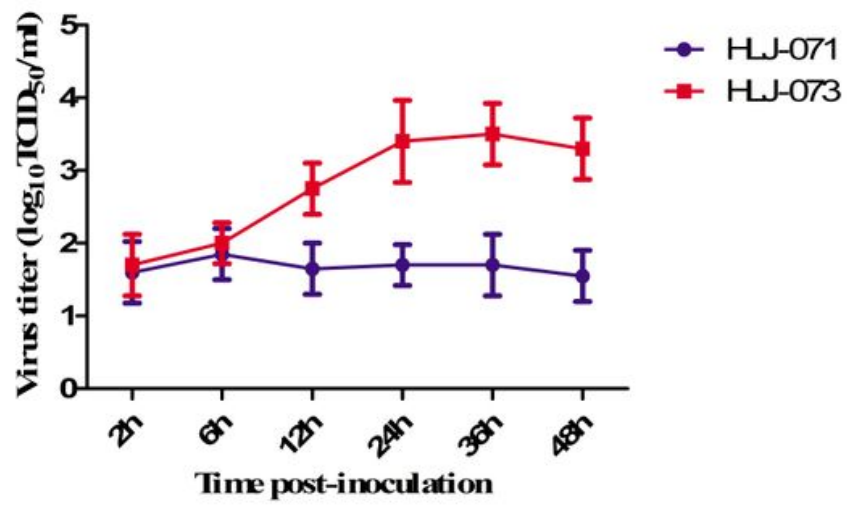

Figure 6

Replication dynamics of HLJ-071 and HLJ-073. Growth kinetics of HLJ-071 and HLJ-073 after infection of $\mathrm{CrFK}(\mathrm{A})$ and canine macrophage/monocyte $(\mathrm{B})$ cells ( $\mathrm{MOI}$ of 1$)$. The titers are given as the means 
from triplicate experiments (log 10 TCID 50/ml); error bars represent standard deviations. 